\title{
Left lobe amoebic liver abscess
}

\author{
RUDRA RASARETNAM \\ M.B., F.R.C.S.
}

\author{
Shelton E. WiJetilaKa \\ M.B., F.R.C.S.
}

\author{
Colombo General Hospital, Ceylon
}

\begin{abstract}
Summary
For reasons which are not quite clear amoebic liver abscesses in the left lobe are relatively uncommon. On account of the lesser bulk of the left lobe, and the potentially greater space under the left hemidiaphragm, expansive lesions within it remain less obtrusive than corresponding lesions in the right. Sixteen such cases are analysed and five distinct modes of clinical presentation have been found. Eight of the patients presented late with perforation into either the peritoneal or pleural cavities. In seven of eight remaining cases, an abdominal mass suggestive of a left lobe lesion was present. Anaemia, leucocytosis and a raised erythrocyte sedimentation rate were usually present, and in the five cases seen in the last year a positive reaction was obtained to the indirect fluorescent antibody test. Confirmatory radiological features were present only in advanced cases.
\end{abstract}

\section{Introduction}

The clinical features of amoebic liver abscesses have been well documented, but most reports have dealt predominantly with lesions in the right lobe (Manson-Bahr, 1932; Ochsner and DeBakey, 1943; Zavala and Hamilton, 1952; Lamont and Pooler, 1958; Paul, 1960). Left lobe lesions are disproportionately less common, accounting for only 10$15 \%$ of all amoebic liver abscesses. Almost the whole of the liver, apart from a small segment in the epigastrium, lies under cover of the lower ribs and costal cartilages, and in the normal individual the liver is not palpable. The early symptoms of hepatic amoebiasis, consisting of pyrexia, malaise, anorexia, abdominal pain, and perhaps chills and rigors, are non-specific, and may occur with many other disease processes. Lesions of the right lobe are recognized clinically by either hepatic enlargement below the costal margin, or by virtue of its impinging on the surrounding structures, mainly the right cusp of the diaphragm and the lower right intercostal spaces. The much smaller size of the left lobe and, consequently, its less obtrusive occupancy of the left upper quarter of the abdominal cavity, together with the rarity of lesions in it, has made difficult the diagnosis of such lesions, resulting in a high incidence of complications.
In this paper, sixteen cases of left lobe amoebic liver abscess who were referred for surgical treatment are analysed with a view to documenting the various clinical syndromes as an aide to earlier diagnosis.

\section{Materials and methods}

The sixteen cases of left lobe amoebic liver abscess are cases who were referred over the past few years for surgical treatment, and do not reflect the true incidence of such lesions. There was the usual male predominance, only two of the patients being female. There was an age range of from 3 months to 74 years, thirteen of the patients being over 40 years of age, including the two females.

The duration of the illness varied from 4 days to 6 months, only seven patients having a history of less than 1 month. Ten patients complained of fever, $50 \%$ of them having an illness of short duration. Abdominal pain was the most consistent symptom, being usually of a constant nature and located in the upper abdomen, but in most cases the patients were rather vague about its character. Only two patients denied a history of abdominal pain, both being cases of rupture into the pleural cavity, although one had experienced left shoulder tip pain at the onset of his symptoms. Anorexia and chest pain were each present in five, four of the latter being patients with pleural rupture. Other symptoms such as chills and rigors, vomiting, dyspnoea, dysphagia and a previous history of blood and mucus per rectum occurred infrequently. Four of the patients were jaundiced, and an epigastric mass or enlarged liver was palpable in nine of the sixteen. Seven patients had an epigastric mass. Left lobe enlargement was present in four while in one other both lobes of the liver were clinically enlarged. Intercostal tenderness was unusual, in marked contrast to patients with right lobe lesions, and even in the two cases in which it was present, it occurred on the right. Four patients presented with massive left pleural effusions due to trans-diaphragmatic rupture. Details of laboratory investigations were not available in all sixteen patients as some had emergency surgery. A polymorphonuclear leucocytosis was common, the total count varying between 9200 and $36,200 / \mathrm{mm}^{3}$; all but one of the patients were clinically anaemic, the 


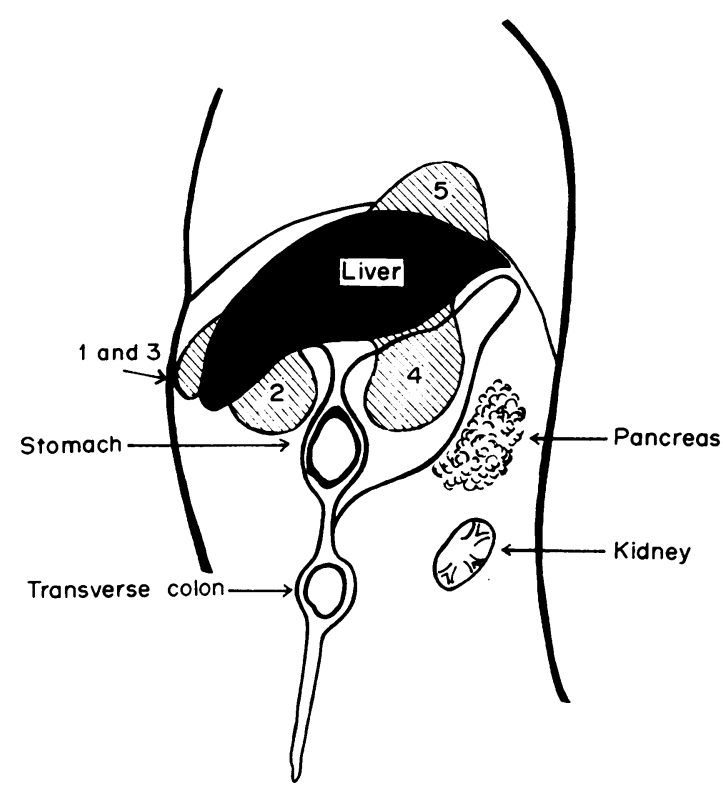

FIG. 1. Diagrammatic representation of the various clinical presentations of left lobe amoebic liver abscesses (see text).

haemoglobin level varying between 4.8 and $11 \cdot 7 \mathrm{~g} \%$. The ESR was consistently raised, with a range of 63-123 mm in the first hour. In the last five patients the indirect fluorescent antibody test was positive in a titre of $1: 128$ or more. In the earlier cases facilities for serological tests were not available.

A retrospective analysis of these cases showed that although the symptoms were vague and non-specific in the majority, five distinct clinical presentations, based mainly on the signs, could be made out. On general examination all the patients looked ill, listless and rather emaciated, and were similar in general appearance to a patient with carcinoma of the stomach. The five clinical syndromes were (Fig. 1):

\section{Type (1)}

Uncomplicated intra-hepatic abscess. Abdominal examination reveals an epigastric mass which is usually tender and moves with respiration. Less commonly the mass is in the left hypochondrium, and in both instances represents an enlarged left lobe of the liver, and is due to an intra-lobar abscess. $\mathrm{X}$-rays are usually negative, and of no help in diagnosis.

\section{Type (2)}

Large hepatic abscess causing gastric compression. Two patients aged 50 and 65 , presented with a vague

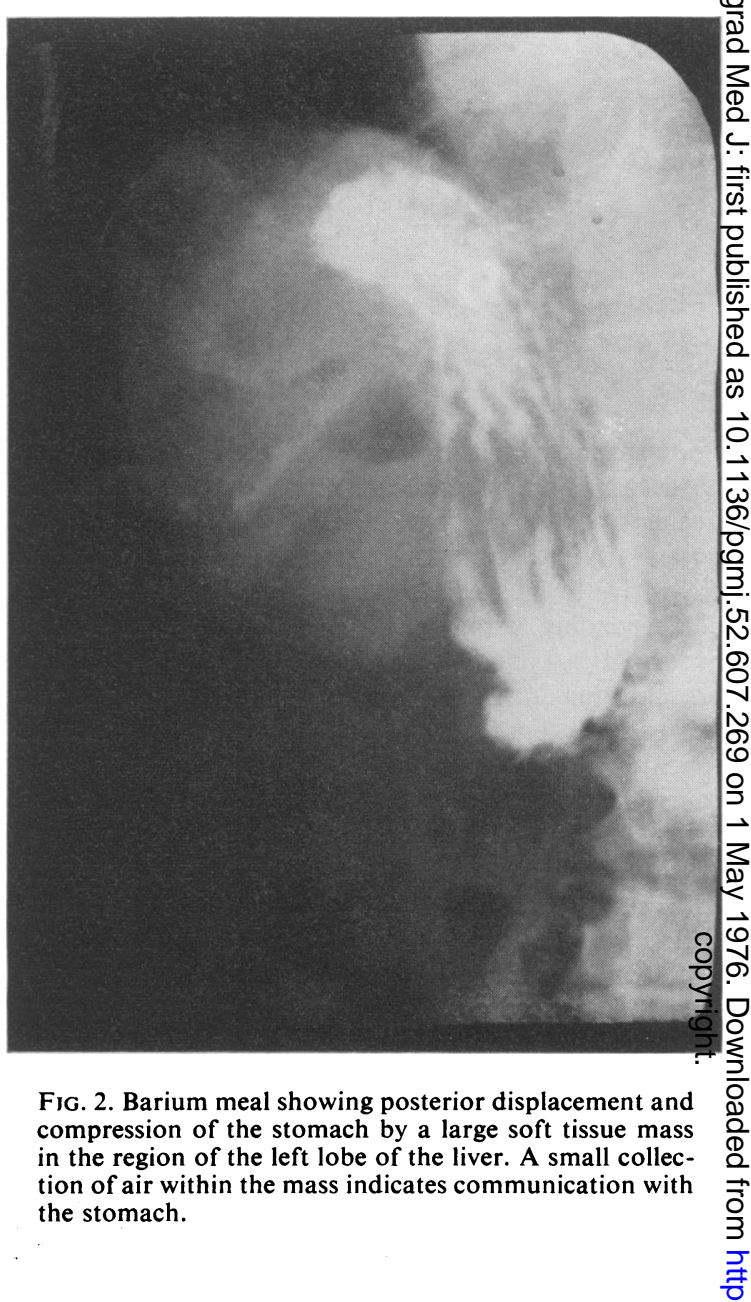

history of illness, each of one month's duration, of upper abdominal pain, anorexia, loss in weight and $\underline{3}$. weakness. One of them also complained of dys phagia, while the other had an irregular pyrexia for a month, and had developed vomiting just before admission. She was mildly jaundiced and had a serum bilirubin of $2.3 \mathrm{mg} \%$. The anorexia in these patientso was more severe than in any of the other types. $\mathrm{No}_{\square}$ abdominal masses were palpable in the former, 을 while in the second patient there was abdominal distension and the liver was palpable one finger's $O$ breadth below the costal margin. The mass in the left $N$ hypochondrium moved slightly with respiration. N Plain X-ray of the abdomen showed the presence of 0 a large soft tissue mass in the left hypochondrium which contained a narrow transverse column of air, $\frac{\mathrm{\Phi}}{\Phi}$ suggesting communication with one of the hollow viscera. A barium meal showed posterior displace- 0 ment of the stomach (Fig. 2) and chest X-ray showedo elevation of the left cusp of the diaphragm with $\mathrm{a}_{\stackrel{\mathbb{Q}}{\mathbb{Q}}}$ 


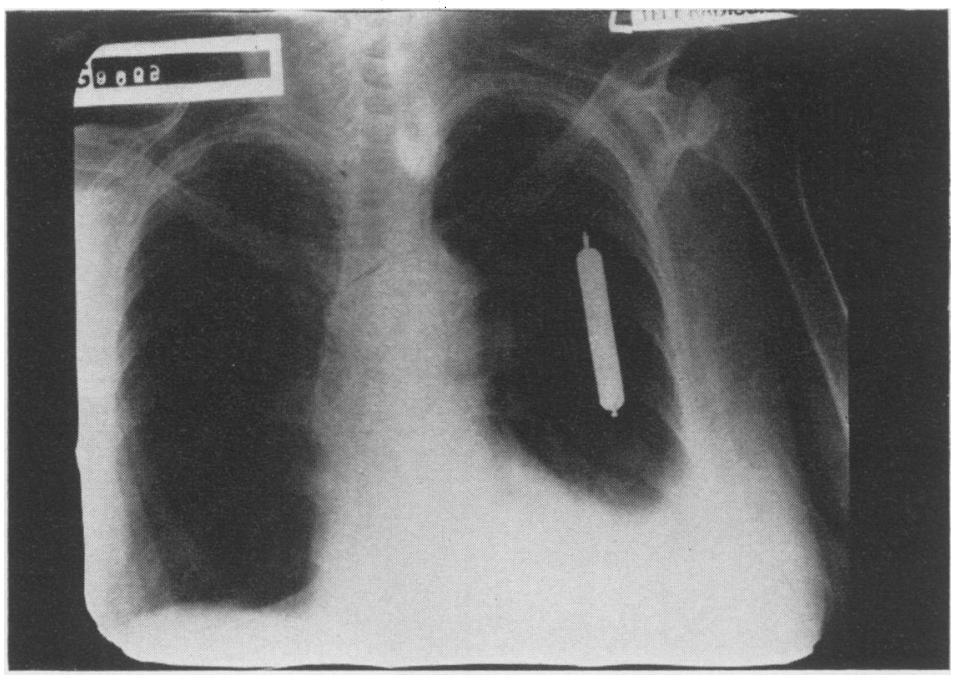

FIG. 3. Chest X-ray: elevation of the left hemidiaphragm and a small pleural effusion occurring as a complication of a left lobe amoebic liver abscess (same patient as in Fig. 2).

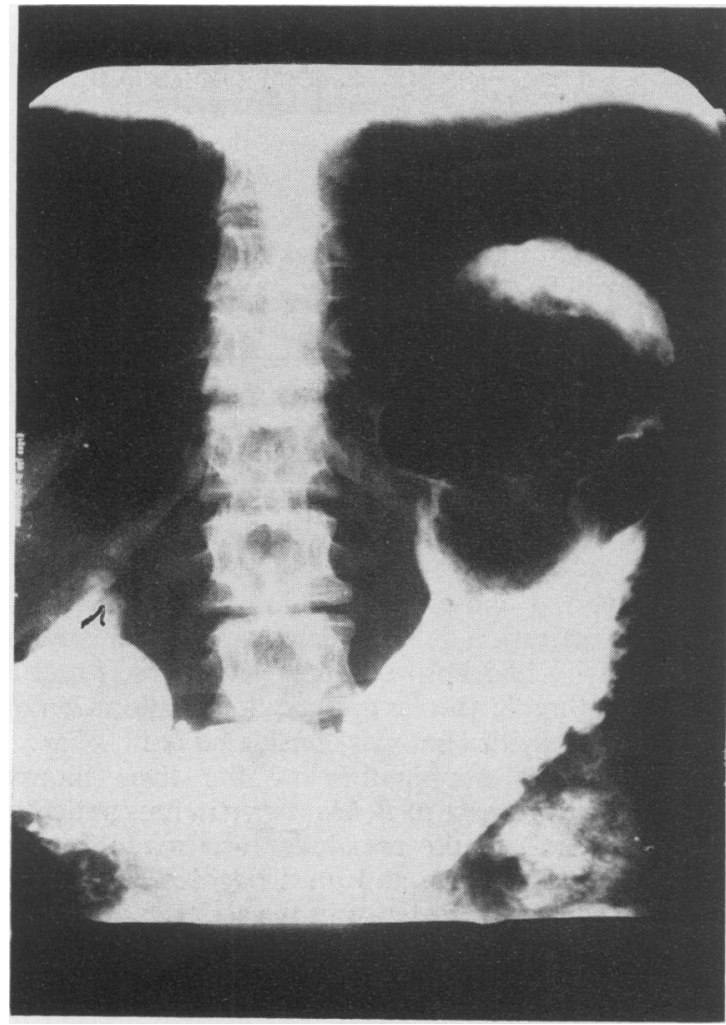

Fig. 4. Barium meal: a large filling defect in the fundus of the stomach caused by a left lobe amoebic liver abscess. small pleural effusion (Fig. 3). In the other case a large filling defect was present in the fundus of the stomach, which was thought pre-operatively to be due to a leiomyoma (Fig. 4). At laparotomy in both cases, a large mass, which was initially mistaken for a tumour, was present in the upper abdomen, but on dissection the 'tumour' suddenly ruptured, liberating a large quantity of typical 'anchovy sauce' pus in which Entamoeba histolytica was identified in one case; in the other, the abscess communicated with the stomach through a small breach in the anterior wall. One of the patients succumbed a week after operation owing to her poor general condition. In both patients, liver biopsy showed the presence of E. histolytica.

Type (3)

Localized intraperitoneal perforation with anterior localization. Two patients presented with a tender epigastric mass which, however, did not move with respiration. The mass represented a small left lobe abscess which had ruptured into the peritoneal cavity, forming a localized abscess between the liver, falciform ligament and the anterior abdominal wall, being walled off by omentum. There were no abnormal X-ray features in these two patients.

\section{Type (4)}

Rupture into the general peritoneal cavity. Three patients had generalized peritonitis due to ruptured left lobe amoebic liver abscesses, one of them being the 3-month-old infant. The history was of less than 1 week in two, while the third had been ill for a month. 


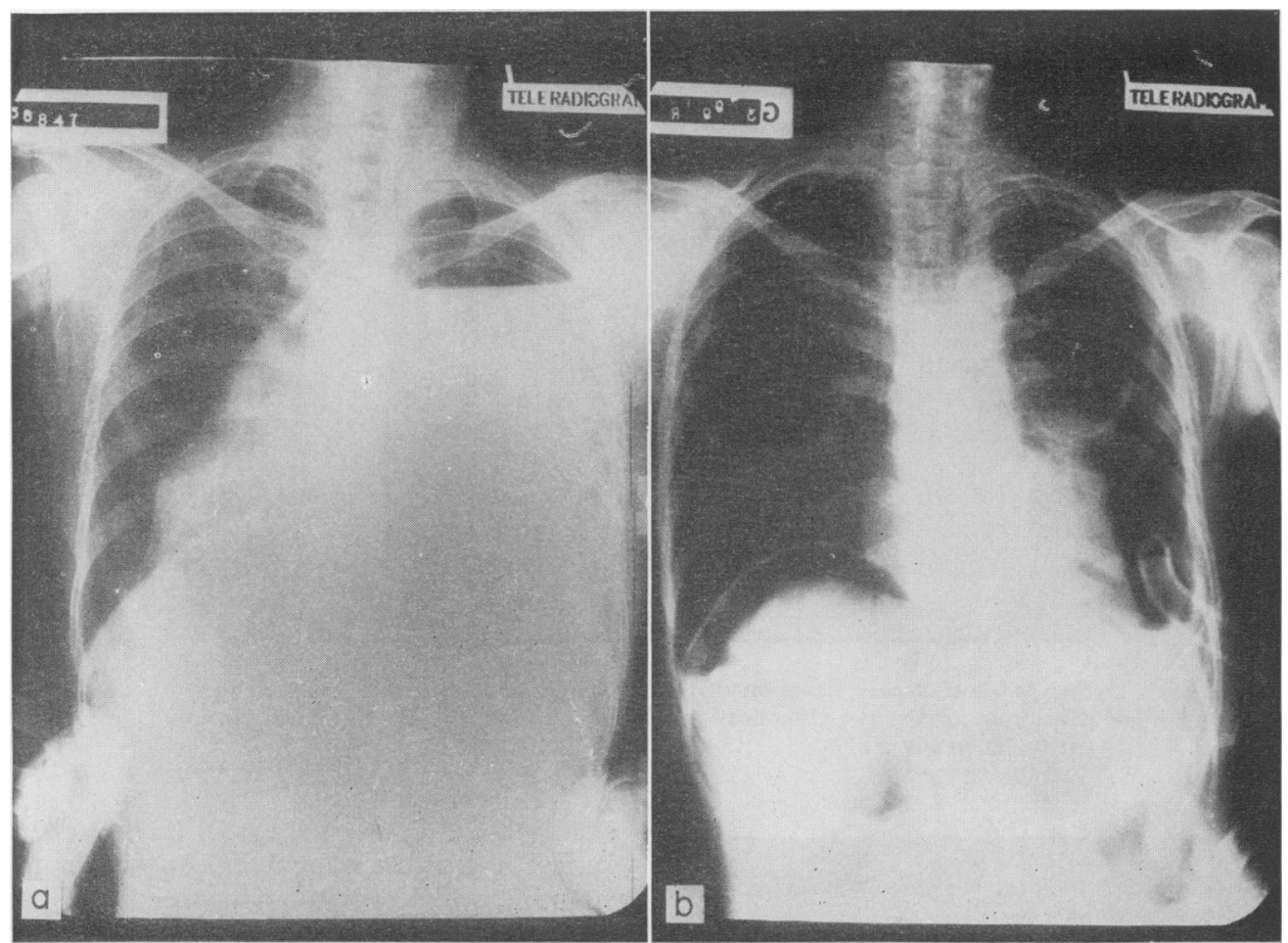

Fig. 5. (a) Intrapleural rupture of a left lobe amoebic liver abscess. (b) Incomplete expansion of the left lung after closed thoracostomy, also showing a right subphrenic abscess.

\section{Type (5)}

Trans-diaphragmatic intrathoracic rupture. The left lobe lies mainly under the central tendon of the diaphragm, and only part of it is in contact with the left hemidiaphragm. Intrathoracic rupture may occur into either the pericardial of left pleural sacs, or directly into the base of the left lung. All four patients presented with empyema, and in one of them there was the subsequent development of a right subphrenic abscess as well (Fig. 5).

\section{Management}

The treatment depended on whether the lesion was still localized or whether it presented as the complication of a liver abscess. Eight of the patients presented in a still localized stage, although the liver abscess in four of them was of considerable size. In Types 1 and 3, mini-laparotomy was performed through a small upper midline incision under local or general anaesthesia and, on confirmation of the diagnosis, the abscess was evacuated and the incision closed without drainage. All four patients thus treated made uneventful recoveries. Two patients (Types 1 and 3) were treated with percutaneous aspiration on one and two occasions respectively, after which both made uneventful recoveries. One other patient underwent formal laparotomy withouta untoward effect.

The two patients who had gastric compression (Type 2) required laparotomy as the diagnosis was윽 obscure in one, and on account of the size of ${ }_{-}$ the abscess in the other. The three patients with generalized peritonitis also required laparotomy two of them succumbing in the immediate postoperative period. The four patients with pleuralempyema were treated initially by closed thoracostomy, and one of them underwent thoracotomyo and decortication later. There was one death: ał patient who had both an empyema thoracis and ao right subphrenic abscess and required exploration of both body cavities but was considered unfit.

Histological examination of the liver biopsy showed the presence of $E$. histolytica in four patients. O The diagnosis in the remainder was made on the $N$ character of the pus, and on a positive serological $\mathbb{W}_{\mathrm{W}}$ test in the last five patients in the series.

\section{Discussion}

Only $10-15 \%$ of amoebic liver abscesses occur in $\stackrel{\oplus}{\oplus}$ the left lobe (Manson-Bahr, 1932; DeBakey and Ochsner 1951; Takaro and Bond 1958). This distribution has been explained on the basis of the lesser $\stackrel{\widetilde{\Phi}}{\mathcal{Q}}$ (a) 
bulk of the left lobe (Shaw, 1949), the greater width and the more linear course of the right branch of the portal vein (Ellsberg, 1906), and the differential distribution of blood in the portal vein depending on its origin from the superior and inferior mesenteric veins. Ghosh (1954) found left lobe liver abscesses when the distal colon was affected by amoebiasis, right lobe lesions being more common with chronic caecal infection.

Right lobe lesions frequently manifest themselves by upward extension on account of the resistance to downward spread by the mass of the lever itself, resulting in pulmonary complications which are recognizable both clinically and radiologically, or by intercostal tenderness when they cause horizontal expansion of the liver, or by localized abdominal tenderness when the liver is enlarged below the costal margin. None of these restricting factors affects the left lobe. Since only a third of the left cusp of the diaphragm is in relationship with the left lobe of the liver, pulmonary symptoms and radiological signs are rare with left lobe abscesses until rupture is imminent (Lamont and Pooler, 1958). The relatively small size of the left lobe and the potentially greater space in the left subcostal area, allow amoebic liver abscesses in the left lobe to remain occult for longer periods than comparable lesions in the right lobe. Added to these factors, the rarity of such lesions has made clinicians less suspicious of their existence until late, resulting in a higher mortality rate (Alkan, Kalmi and Kalderon, 1961).

Laboratory tests have not until recently been of diagnostic importance. The introduction of serological tests in the diagnosis of hepatic amoebiasis, and the high incidence of positive results in the presence of such lesions, makes them useful confirmatory tests when the diagnosis is suspected. In this small series, the indirect fluorescent antibody test was positive in all five patients in whom it was carried out.

As an aide to diagnosis, five modes of clinical presentation are documented. The only symptom that was nearly always present was abdominal pain, and rather surprisingly only $60 \%$ gave a history of pyrexia. It was lacking equally in short duration illnesses of only a few days, as in patients with a history of 6 months. Evidence of an expansive lesion in the left lobe may easily escape detection under the umbrella of the left lower ribs, but it is curious that while quite small abscesses may rupture, giving rise to a generalized peritonitis, the lesion may expand to the size of a grapefruit and yet remain intrahepatic, although gastric compression would occur in these latter cases. Presumably, these differences in tissue reactions are due to variations in host resistance. Soft tissue abdominal masses may be seen radiologically in such cases, and even elevation of the left hemidiaphragm, basal atalectasis or pleural effusions in the left costo-phrenic angle (Alkan et al., 1961; Ramachandran, 1974). Barium study would confirm the presence of gastric compression which may occur from in front as in the cases in this series, or from the lesser sac posteriorly, as in the cases described by Alkan et al.

Concomitant enlargement of the right lobe of the liver occurs in some cases, as happened in one patient in whom just over 3 litres of typical amoebic pus were aspirated from the left lobe. The right hepatomegaly is neither tender nor shows localizing signs, and its rapid diminution in size with aspiration of the left lobe abscess confirms the view of Lamont and Pooler (1958) that it is caused by reflex vascular dilatation of the hepatic veins.

Amoebic liver abscesses require aspiration and/or decompression if there is no response to amoebicidal drugs within 5 days, or if there is deterioration in the general condition, or if rupture is imminent. The abscess in three of the four patients who died had already ruptured at the time of admission, and that in the fourth had formed a communication with the stomach. Unlike direct aspiration of the right lobe through the intercostal spaces, percutaneous aspiration of the left lobe through the anterior abdominal wall is not without risk, mainly on account of the possibility of errors in diagnosis. The procedure of mini-laparotomy is both simple and safe; an upper midline incision 3-4 cm long is carried through the linea alba into the peritoneal cavity. The bulging smooth surface of the left lobe abscess is now seen and may be aspirated through a 15 gauge needle with complete safety. Where localized extrahepatic collections have formed, drainage is obtained as the incision is carried through the abdominal wall. This procedure obviates the risk of percutaneous aspiration when tumours of the liver, stomach or pancreas may be mistaken for left lobe liver abscesses. The unusual large left lobe abscesses causing gastric compression, and patients with generalized peritonitis are best treated by formal laparotomy. Pleural or pericardial collections require tube drainage as an initial measure, and may later require thoracotomy.

\section{Acknowledgments}

We have pleasure in thanking Miss Nelun Fonseka for the diagram, and Mr Karunasena Gomes for the figures.

\section{References}

Alkan, W.J., Kalmi, B. \& Kalderon, M. (1961) The clinical syndrome of amebic abscess of the left lobe of the liver. Annals of Internal Medicine, 55, 800.

DeBAKEY, M.E. \& OCHSNER, A. (1951) Hepatic amebiasis. Surgery, Gynecology and Obstetrics. International Abstracts of Surgery, 92, 209.

EllsBerg, C.A. (1906) Solitary abscess of the liver. Annals of Surgery, 44, 217. 
Gновн, B.C. (1954) Tropical liver abscess affecting the left lobe. Indian Medical Gazette, 89, 152.

Lamont, N. McE. \& Pooler, N.B. (1958) Hepatic amoebiasis. A study of 250 cases. Quarterly Journal of Medicine, 27, 389.

MANSON-BAHR, P. (1932) Amoebic abscess of the liver; its diagnosis and treatment. A clinical study. Proceedings of the Royal Society of Medicine, 25, 233.

OChSNer, A. \& DeBAKEY, M.E. (1943) Amebic hepatitis and hepatic abscess. An analysis of 181 cases with review of the literature. Surgery. St Louis, etc., 13, 460 \& 613.

PAUL, M. (1960) New concepts on amoebic abscess of the liver. British Journal of Surgery, 47, 502.
RAMACHANDRAN, S. (1974) Radiological changes in left lobe ֶ amoebic liver abscesses. Postgraduate Medical Journal, 50, 689.

SHAw, R.R. (1949) Thoracic complications of amebiasis. Surgery, Gynecology and Obstetrics, 88, 753.

TAKaro, T. \& Bond, W.M. (1958) Pleuro-pulmonary, pericardial and cerebral complications of amebiasis. Surgery. Gynecology and Obstetrics. International Abstracts of Surgery, 107, 209.

ZaVALA, D.C. \& Hamilton, H.E. (1952) Recognition and $\frac{\bar{\omega}}{\nabla}$ treatment of hepatic amebiasis. Annals of Internal Medi- $\varrho$ cine, 36, 110. 